\title{
La reconstruction des maxillaires
}

\section{Tulasne JF}

26, avenue Kléber, Paris, France

jftulasne@wanadoo.fr

Les indications de reconstruction du maxillaire et de la mandibule se sont multipliées avec l'essor de l'implantologie. L'utilisation d'un os cortical autogène, donc de densité élevée, permet de limiter la résorption des greffons et d'obtenir une masse osseuse de volume suffisant pour placer des implants, la forte densité assurant un excellent ancrage à des implants même très courts.

La voûte crânienne est le meilleur site donneur car l'os (cortical d'origine membraneuse) y est le plus souvent abondant et les suites opératoires sont les plus simples qui soient (absence habituelle de douleur et d'œdème, aucune cicatrice visible), et le comblement de la zone de prélèvement par une résine acrylique (utilisée en orthopédie pour sceller les prothèses de hanche) évite toute déformation et toute fragilité de la voûte crânienne.

Dans certaines situations bien particulières, il est possible d'utiliser des biomatériaux qui peuvent constituer une alternative plus simple pour le patient et le chirurgien. 